against targets not tested for in clinic. In this study, we aimed at extending the detection of autoantibodies by including all cytoplasmic aaRS in the analysis of patients with IIM. We hypothesized the existence of new potential autoantigens within this protein family.

Methods: The presence of anti-aaRS autoantibodies was determined using a multiplex suspension bead array assay on 242 IIM patients from the Karolinska University Hospital myositis cohort. A panel of 186 recombinant constructs, representing 57 proteins that included full-length or partial sequence overlaps between constructs of all cytoplasmic aaRS as well as other myositis related proteins, were coupled to magnetic color-coded beads and each plasma sample was tested against the complete antigen panel.

Results: By the use of this multiplex method we identified patients with autoantibodies against many of the tested aaRS. Autoantibodies binding to HisRS have previously been shown to bind with higher reactivity to the WHEP domain of HisRS and this was also confirmed in this study. We confirmed reactivity against three of the other aaRS tested for in the clinic (PL-12, PL-7, and EJ). In addition, we identified patients positive for anti-Zo, -KS and -HA, autoantibodies usually not screened for in routine. Finally, our data indicates that there are autoantibodies binding to other aaRS than the previously known eight autoantigens, which will be presented.

Conclusion: In this study, we could detect autoantibodies in plasma from patients with IIM, both against the most common aaRS autoantigens, but also against other aaRS that are usually not tested for in clinic. We conclude that it is important to continue the studies of anti-aaRS autoantibodies, and their correlation to clinical manifestations, and in the long run also include more aaRS autoantigens in clinical practice.

References:

[1] Dobloug, G.C., et al., Mortality in idiopathic inflammatory myopathy: results from a Swedish nationwide population-based cohort study. Ann Rheum Dis, 2018. 77(1): p. 40-47.

[2] Barsotti, S. and I.E. Lundberg, Myositis an evolving spectrum of disease. Immunol Med, 2018. 41(2): p. 46-54.

[3] Vencovsky, J., H. Alexanderson, and I.E. Lundberg, Idiopathic Inflammatory Myopathies. Rheum Dis Clin North Am, 2019. 45(4): p. 569-581.

[4] Richards, T.J., et al., Characterization and peripheral blood biomarker assessment of anti-Jo-1 antibody-positive interstitial lung disease. Arthritis Rheum, 2009. 60(7): p. 2183-92.

Disclosure of Interests: Charlotta Preger: None declared, Antonella Notarnicola: None declared, Cecilia Hellström: None declared, Edvard Wigren: None declared, Catia Cerqueira: None declared, Peter Nilsson: None declared, Ingrid E. Lundberg Grant/research support from: Bristol Meyer Squibb, Corbus Pharmaceuticals, Inc and Astra Zeneca, Helena Persson: None declared, Susanne Gräslund: None declared, Per-Johan Jakobsson Shareholder of: Gesynta Pharma, Grant/research support from: Gesynta Pharma, AstraZeneca,

DOI: 10.1136/annrheumdis-2020-eular.1414

\section{SAT0289 THE INS AND OUTS OF EPIDERMAL DYSFUNCTION IN SYSTEMIC SCLEROSIS (SSC): RESULTS FROM A NOVEL TISSUE ENGINEERED EPIDERMAL EQUIVALENT FROM SSC KERATINOCYTES}

B. Russo ${ }^{1}$, J. Borowczyk-Michalowska ${ }^{1,2}$, W. H. Boehncke ${ }^{2}$, N. Brembilla ${ }^{1,2}$, C. Chizzolini'. ${ }^{1}$ Pathology \& Immunology, School of Medicine, University of Geneva, Geneva, Switzerland; ${ }^{2}$ Department of Dermatology, University Hospital and School of Medicine, University of Geneva, Geneva, Switzerland

Background: Skin fibrosis is a hallmark of systemic sclerosis (SSc). It is commonly accepted that vascular damage, immune system activation and, abnormal fibroblasts-to-myofibroblasts differentiation are pathological capital features. Nevertheless, recent evidence portrays a potential role of the epidermis in the pathogenesis of SSc skin fibrosis $(1,2)$. This new angle on skin fibrosis pathogenesis is particularly attractive as the epidermis is an easy to access therapeutic target. Objectives: To dissect the potential epidermal dysfunction in SSc and its effect on dermis homeostasis, using a novel epidermal equivalent reconstituted from SSc keratinocytes.

Methods: Primary keratinocytes and fibroblasts cell lines were generated from skin biopsies obtained from 6 SSc and 6 healthy donors (HD), upon informed consent and ethical approval. Epidermal equivalents (EE) were generated from 4 SSc and $6 \mathrm{HD}$ keratinocytes. Skin and EE expression of the mitotic marker Ki67, of the differentiation markers (K10, involucrin, filaggrin, loricrin), and activation markers (K6, K16) was evaluated by immunohistochemistry. The transcriptomic profile of SSc keratinocytes in monolayer or stratified in EE was identified by RNAseq analysis. EE conditioned medium was used to stimulate fibroblasts. The fibroblast production of interleukin (IL)-6, IL-8, matrix metalloproteinase (MMP)-1, type-I collagen (col-I), and fibronectin was assessed by ELISA.

Results: Compared to HD, immunohistochemistry revealed that SSc epidermis is characterized by aberrant premature differentiation and enhanced expression of activation markers associated with a lower mitotic rate of basal keratinocytes. Of interest, EE reconstituted from SSc keratinocytes reproduced most of the abnormalities observed in SSc epidermis. RNAseq analysis revealed that SSc keratinocytes, either cultured in monolayer or in $\mathrm{EE}$, have a distinct transcriptomic profile compared to their HD counterpart, characterized by the downregulation of genes from the HOX family.

The supernatant of EE enhanced the production of IL-6, IL-8, MMP-1, col-I, and fibronectin by HD fibroblasts $(p<0.05)$. Except for col-I and fibronectin, this effect was 2-fold higher in the presence of supernatant from EE reconstituted by SSc keratinocytes. Neutralization experiments indicated that IL-1 was, at least in part, responsible for keratinocyte-dependent fibroblasts activation.

Conclusion: We established a novel epidermal equivalent tissue engineered from SSc keratinocytes, that recapitulates the in vivo characteristics of SSc epidermis. Our preliminary data suggest that SSc keratinocytes have an intrinsic altered program of differentiation, possibly due to the downregulation of some HOX genes. This altered phenotype is associated with increased production of mediators that stimulate fibroblasts production of inflammatory cytokines. In this scenario, we may hypothesize that SSc epidermis participates in modifying the dermis environment, favoring the development of chronic inflammation and fibrosis.

\section{References:}

[1]Takahashi T, Asano Y, Sugawara K, Yamashita T, Nakamura K, Saigusa R, et al. Epithelial Fli1 deficiency drives systemic autoimmunity and fibrosis: Possible roles in scleroderma. J Exp Med. 20317;214(4):1129-51.

[2] Nikitorowicz-Buniak J, Shiwen X, Denton CP, Abraham D, Stratton R. Abnormally differentiating keratinocytes in the epidermis of systemic sclerosis patients show enhanced secretion of CCN2 and S100A9. J Invest Dermatol. 2014;134(11):2693-702.

Disclosure of Interests: Barbara Russo: None declared, Julia Borowczyk-Michalowska: None declared, Wolf-Henning Boehncke Consultant of: WHB received honoraria as advisor or invited speaker from Abbvie, Almirall, BMS, Celgene, Leo, Lilly, Novartis, UCB., Speakers bureau: WHB received honoraria as advisor or invited speaker from Abbvie, Almirall, BMS, Celgene, Leo, Lilly, Novartis, UCB. Nicolò Brembilla: None declared, Carlo Chizzolini Consultant of: Boehringer Ingelheim, Roche

DOI: 10.1136/annrheumdis-2020-eular.2214

\section{SAT0290 \\ HIGH SERUM MYOSTATIN LEVELS SUGGEST ACCELERATED MUSCLE SENESCENCE IN ACTIVE IDIOPATHIC INFLAMMATORY MYOSITIS}

\section{A. Anuja ${ }^{1}$, M. Singh ${ }^{1}$, M. K. Rai ${ }^{1}$, H. Singh ${ }^{1}$, V. Agarwal ${ }^{1}$, L. Gupta ${ }^{1}{ }^{1}$ Sanjay Gandhi Postgraduate Institute of Medical Sciences, Lucknow, India}

Background: Inflammation is the forerunner to fibrosis and premature ageing in various systemic diseases. Hence it seems plausible that idiopathic inflammatory myopathies (IIM) may exhibit accelerated senescence too.

Objectives: Hence we investigated the Myostatin: Follistatin system in the serum as a reflection of early senescence in myositis as compared with healthy and diseased controls

Methods: Patients with inflammatory myositis (ACR/EULAR criteria) presenting to the wards and outpatient clinic between December 2017 to August 2019 were recruited. Those with active infection, pregnancy, renal dysfunction or chronic kidney disease were excluded. Apart from patient and disease variables, activity and damage were assessed using standard IMACS score set measures. Patients in inception cohort were additionally followed up at 1 and 6 months. Myostatin and Follistatin were estimated in sera using ELISA (R\&D systems, USA). Juvenile myositis and young adults (18-40 years) were subsequently analyzed separately. Non-parametric tests were used for paired and unpaired analysis. Results expressed as median.

Results: 95 myositis (8 Juvenile myositis, 26 DM, 10 PM, 29 Overlap, 2 NAM 1 CAM and 19 ASS) patients (23 Male and 72 Female) with median age 38 (24.5-46.0) years and disease duration 0.9 (2.3-5.1) years were included. Serum Myostatin was lower in IIM than in healthy control (HC) (153.5 vs. $243.6 \mathrm{p}<0.0001$ Fig $1 \mathrm{~A}$ ) but higher in IIM as compared with disease controls (153.5 vs 86.1 $\mathrm{p}=0.0174$ Fig. 1B). Serum myostatin was comparable between juvenile and adult myositis and in the various subsets of adult myositis (Fig. $1 \mathrm{C}$ and D). Myostatin levels were higher in active as compared with inactive myositis in young adults (211.7 vs. 158.9, $p=0.0149$, Figure $1 \mathrm{E}$ ). Serum Myostatin correlated with height ( $r 0.3, p=0.003)$ and weight $(r 0.2, p=0.047)$ but not MMT8 or muscle enzymes. 